\title{
Using Antenna Patterns to Improve the Quality of SeaSonde HF Radar Surface Current Maps
}

\author{
Donald E. Barrick and Belinda J. Lipa \\ CODAR Ocean Sensors \\ 1000 Fremont Avenue, Suite K \\ Los Altos, CA 94024
}

\begin{abstract}
The SeaSonde coastal HF current mapping radar realizes its very small and convenient antenna size by employing the MUSIC direction finding (DF) algorithm, rather than beam forming to determine the bearing angle to each point on the sea plot. Beam forming requires large phased array antennas spanning up to $100 \mathrm{~m}$ of linear coastal extent. The SeaSonde uses two colocated crossed loops and an omnidirectional monopole as the receive antenna system. Unique features of DF polar maps can be angle sectors with sparse coverage or gaps. This occurs when the antenna patterns are distorted by nearby terrain or buildings, a frequent effect observed with all HF radars. In addition, uncorrected distortions can produce angle biases (misplacements) of the radial current vectors, sometimes as much as $10^{\circ}$.

The actual antenna patterns (with distortions) are now measured with a small battery operated transponder, either from land in front of the antenna or from a boat. These are then inserted into the software to calibrate, i.e., correct for the distortions. A number of algorithms have been evaluated for this correction process, both using simulations (with known input) as well as actual measured sea echo. We present the latest findings on gap and bias mitigation, which show that nearly all of these deleterious effects can be reduced to acceptable levels.
\end{abstract}

\section{INTRODUCTION}

Surface current maps from coastal HF radars are generally synthesized by combining radially directed velocity vector maps in polar coordinates, as measured by two spatially separated sites. The greatest source of bias in such maps traces to errors in the bearing angles associated with the radial velocities. Determination of the radar echo bearing depends on knowledge of the relation between the receive antenna element voltages and their radiation patterns. Initially, HF radar users assumed these antenna patterns were the perfect "textbook" predictions, with ideally balanced channel/cable amplitudes and phases. Measurements over the past several years have revealed that this is rarely the case: patterns are distorted by nearby environmental irregulatities. CODAR Ocean Sensors
(COS) has developed and employs small battery-operated transponders that are used to measure the receive antenna patterns: either on land in front of the antenna or from a boat.

The irregularities that distort patterns are larger than a quarter of a wavelength and located within 1-2 wavelengths of the antenna. At the lower HF bands used (e.g., 12-14 MHz), these distances are $\sim 25-50 \mathrm{~m}$, reducing proportionately at the upper bands $(45-50 \mathrm{MHz})$ to a quarter of this amount. These distortions depend on the antenna environment, and not on manufacturing imperfections in the antenna itself. This has been verified by replacing one antenna unit by another at the same location and remeasuring, to obtain nearly the same pattern. Conversely, patterns measured as part of manufacturing quality control on idealized antenna ranges tend to be perfect, but then become distorted when installed at the coast. Among the features known to distort the patterns are nearby posts, buildings, metal fences, trees, power lines, layout of the building if rooftop mounting is necessary, dunes or terrain ridges under the antenna, proximity to the edge of a cliff or sharp slope, and inadequate isolation of the feedlines running to the antenna elements.

We have devoted considerable effort investigating how best to employ these measured patterns in calibrating and correcting for effects of the distortions. With the COS direction-finding algorithms (i.e., MUSIC, [1], and leastsquares, [2]), these patterns are used directly in models of the antenna signal covariances. Our first such attempts produced gaps (angle sectors where radial vectors were sparse) in certain directions. As a result, we continued to recommend using 'perfect patterns' (although the measured patterns were not perfect), because the latter yielded more uniform coverage. Closer examination (e.g., Emery and Washburn, [3], and Melton [4] showed bearing biases up to $10^{\circ}$ when pattern distortions were ignored in this way, i.e., measurements reveal that radial vectors were misplaced consistently in a given direction by this amount. This bias changes from point to point on the map, so the entire polar map of radial current vectors cannot merely be rotated by a fixed amount to correct for it.

We confirm this observation by simulations, where the input current patterns are known and serve as the standard for 
comparison. Our results agree with those of Emery and Washburn [3]. We show that when the measured patterns are used in the extraction processing -- instead of assuming them perfect -- these bearing biases disappear. However, small bearing gaps. i.e., regions with sparser coverage, now appear. We discuss the implications of this effect and some remedies.

\section{GAPS IN COVERAGE BASED ON SIMULATIONS WHEN ANTENNA PATTERNS ARE DISTORTED}

Simulations here imply the use of artificially generated sea echo data as a basis to test current extraction algorithms [5]. These algorithms incorporate the Gaussian random nature of the Bragg-scattering ocean waves, and thence the radar signals. They allow the inclusion of user-selected short-wave distributions with angle, which define the variances of the zero-mean Gaussian signals scattered from the sea vs bearing angle. These echo signals -- as Doppler shifted by the wave and current velocities -- comprise the Bragg spectral peaks. The user also specifies the radial surface current distribution vs bearing angle and range from the radar. Randomness can be added to these mean input current pattern distributions -- for each bearing and range cell -- to simulate natural turbulence or variations.

The rationale for why one uses simulated data to study problems and develop solutions for them is that one knows precisely the input with which to "ground-truth" the output. This procedure has advantages over actual field tests, for which ground truth is generally costly, limited in quantity, and from instruments that measure something different in nature from the $H F$ radar surface currents.

Other radar-specific parameters are also set, including the radar frequency and range cell width. Most important, the antenna patterns for each of the receive elements are included. These are typically the distorted patterns that are measured with the transponder for one of the SeaSonde sites. Often for comparison we use perfect, idealized patterns to obtain a feeling for how distorted patterns must be in order to bias the output significantly. The files outputted from the simulation program are in the form of raw cross spectra, in exactly the same format as actual data acquired by the SeaSonde, so that the same algorithms used in the field can be used to process these data files.

We simulated an actual SeaSonde scenario where data have been collected for over a year by the University of Santa Barbara (CA). The particular radar examined is located on the campus itself, looking South into the Santa Barbara Channel. The system operates at $13.47 \mathrm{MHz}$ and uses the normal SeaSonde colocated crossed-loop/monopole receive antenna unit. Patterns were measured with the transponder during January 1998.

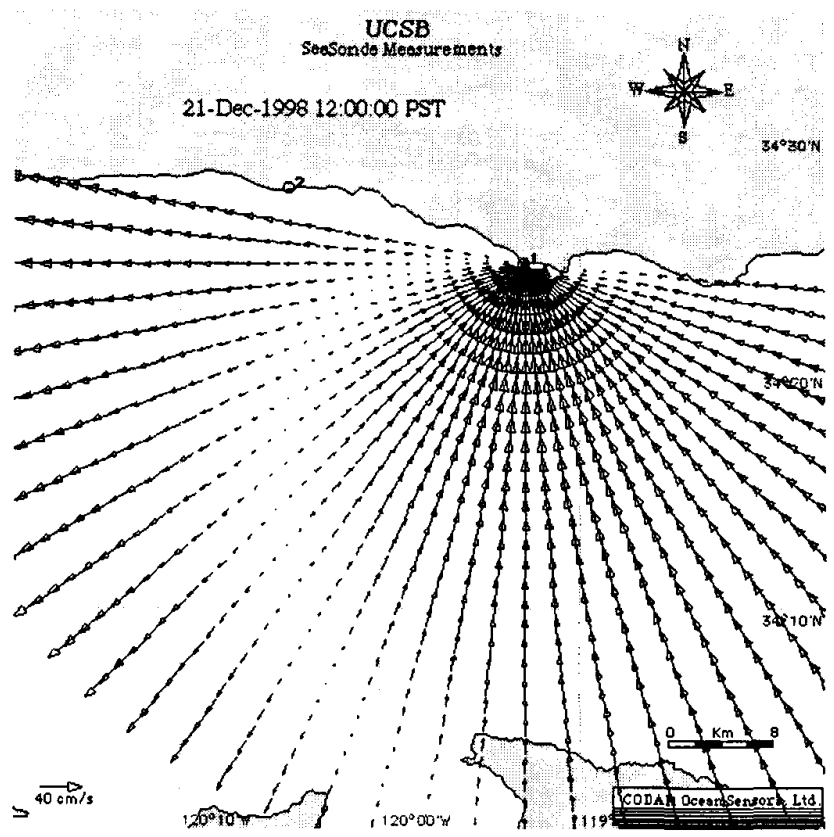

Fig. I One sample of input mean surface current radial velocity map used in simulations for antenna pattern bias studies.

A variety of radial velocity current patterns and Braggscattering wind-wave patterns were generated as inputs to the simulations. An example of one such mean radial current pattern is shown in Fig. 1. To this mean pattern, a zero-mean Gaussian randomness with a standard deviation of $5 \mathrm{~cm} / \mathrm{s}$ is added to each point on the map. A total of 124 range-cell samples were used in the study.

The actual distorted antenna patterns we measured were used to generate the simulated radar spectra. These normalized antenna patterns are shown in Fig. 2. The polar plots are the absolute value of the ratio of the two crossed loop patterns divided by the monopole pattern. The points are the individual values measured by the boat, while the heavy lines through them are the mean fit to the points. The ' $x$ ' symbols and heavy mean line represent the Loop \#1 ratio, while the ' $o$ ' symbols and heavy dashed line represent Loop \#2. Idealized (perfect) patterns are shown as the solid and dashed cosine/sine curves for reference. These are typical of more moderately distorted patterns we encounter. In this case, the receive antenna is at the edge of a cliff with trees and power lines very close by.

First we studied the coverage density of retrieved radial current velocity vectors found vs bearing angle, over the same sector that antenna patterns were measured. In the past (before antenna pattern measurements were made and used), ideal patterns were assumed in the standard MUSIC DF retrieval process; this is the processing used in the measurement comparisons by Emery and Washburn [3]. When we used 
ideal patterns to recover the radial current velocities based on our simulated, we found that the coverage density of the radial vectors with bearing angle was nearly uniform. We do not show this result here. However, when we used the measured patterns (the same ones as used to generate the input radar spectra), we obtain the coverage density vs bearing angle shown as the shaded annulus of Fig. 2. Although one might think that this should produce better output data than the incorrect use of idealized patterns, one sees narrow angle sectors of lower density vector coverage. Closer inspection shows these "gappy" sectors to coincide with bearings having "bumps" in the antenna patterns, i.e., greater departures from the ideal shape shown. This coincidence suggests that the DF algorithm has difficulties obtaining a solution where the pattern change vs bearing is the greatest; the reason for this is not known at present.

Uniform coverage is desired, and one might be led to believe that this, therefore, is an indicator of quality and/or accuracy of the output. The next section shows that this is not the case.

\section{ANGLE BIASES OF RADIAL VECTORS WHEN ANTENNA PATTERNS ARE DISTORTED}

Emery and Washburn [3] studied biases by examining the long-term time correlation between radial currents extracted by the SeaSonde MUSIC DF algorithm and the radial component of subsurface current measured by a moored ADCP at a bearing $191^{\circ} \mathrm{T}$ from the radar site. The ADCP bin 3 meters below the surface was used. The HF radar feels the current in the upper meter of water. Correlation coefficients

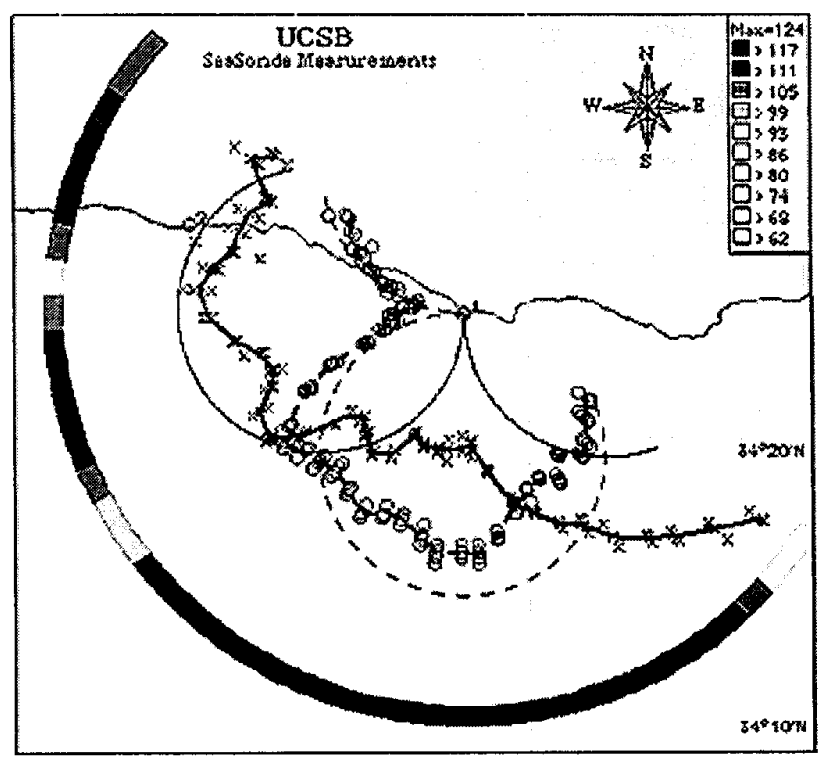

Fig. 2. Polar plot of measured antenna patterns: crossed loop voltage signals normalized to monopole signal. Perfect sine/cosine patterns shown for reference. Annular ring shows density of retrieved radial current vectors vs angle. were computed between the ADCP (at its fixed bearing) and radar speeds at all bearing angles. If there were no bias, the correlation coefficient should be highest for the radar bearing that coincides with the ADCP bearing. They found slightly higher correlation about $10^{\circ}$ counterclockwise, i.e., about $180^{\circ} \mathrm{T}$. The correlations were about $66 \%$ at $191^{\circ} \mathrm{T}$ vs $72 \%$ at $\sim 180^{\circ} \mathrm{T}$. More than 1200 hourly samples over a four-month period were used, and ideal (perfect) patterns were assumed in the extraction process. Therefore, one concludes that the output at that location was biased in bearing by about $10^{\circ}$.

We simulate this situation here by comparing the extracted radial currents from the simulated radar data for all bearings with the input mean radial current data along $191^{\circ} \mathrm{T}$, the same bearing as the Santa Barbara ADCP. Both simulated and measured echo data ought to be biased in the same way by the distorted antenna pattern. First, we looked at the correlation coefficients. These are plotted in the upper half of Fig. 3.
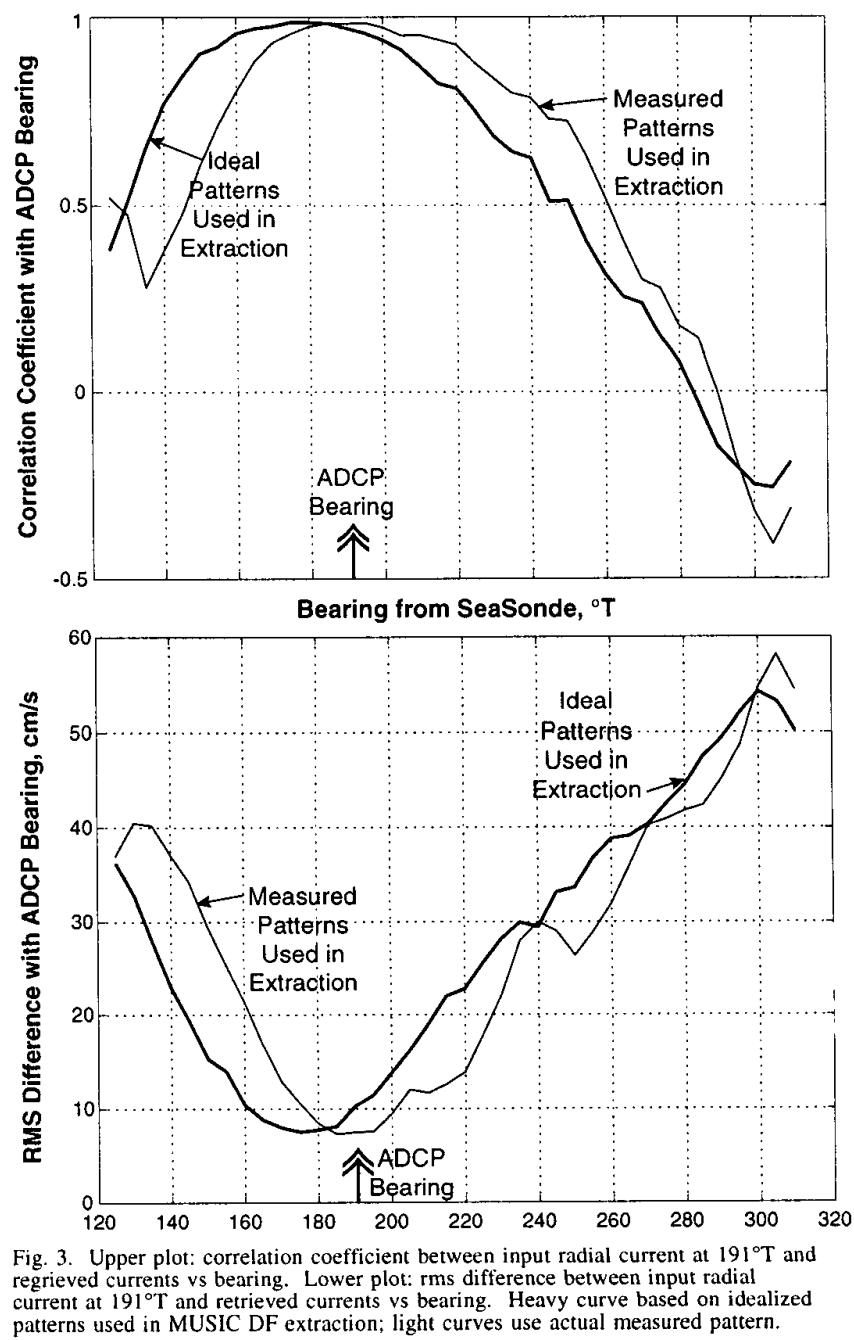
The solid curve is the correlation coefficient when the idealized patterns are used in the MUSIC DF extraction process; the light curve represents the use of the actual distorted pattern itself during extraction. In agreement with measurements [3], the peak correlation when ideal patterns are used occurs about $10^{\circ}$ counterclockwise from the truth. On the other hand, measured patterns produce the best correlation at the ADCP bearing near $191^{\circ} \mathrm{T}$.

As a second check, we compared the root-mean-squared (rms) difference between input radial currents at bearing $191^{\circ} \mathrm{T}$ and extracted currents at all bearings. Both ideal and measured distorted patterns were again used in the extraction process. The curves are shown in the bottom half of Fig. 3. We find the same results as we obtained from the correlation study. When measured patterns are used, lowest rms differences occur where they should, at $191^{\circ} \mathrm{T}$, while use of the ideal patterns shows a bias about $10^{\circ}-15^{\circ}$ counterclockwise.

\section{DISCUSSION AND CONCLUSIONS}

It seems reasonable that correct use of antenna patterns should produce less biases or errors in output radial current maps. Until recently, we had been concerned over whether the patterns themselves were being measured correctly. Within the past year this concern was resolved as pattern repeatability has been verified by measurements at different times (e.g., days apart) and ranges from the radar.

Our results reveal two things. If one ignores the distortions by using ideal patterns in the extraction process, he will produce more pleasingly, uniformly dense distributions of radial velocity fields with bearing angle. Use of patterns tends to show narrow angle sectors $\left(5^{\circ}-15^{\circ}\right)$ with sparser coverage.

However, placement of the radial current vectors can be biased as much as $10^{\circ}$ in bearing angle when ideal patterns are inappropriately used. The biases we find based on simulations are confirmed by field measurements for the same antenna patterns. Employing the correct measured patterns with the distortions appears to eliminate these biases for the cases we have studied.

From our limited case studies thus far, it also appears that a $10^{\circ}$ bias at one location -- when ideal patterns are used -- is not constant across the map. It tends to vary from point to point; this will be studied later. Thus, one cannot simply rotate the entire current vector map by a bias amount that is found at one bearing.

We therefoe recommend using the measured antenna patterns in the extraction process. In the gap regions where solution density is sparser, we find that the fewer retrieved velocities are no less accurate than elsewhere (compared to known input). Where there are narrow sectors with no solutions at all, we recommend interpolation in bearing across gaps up to $15^{\circ}-20^{\circ}$. Beyond this, interpolated values become questionable, and perhaps it is more prudent to leave the gaps.

\section{REFERENCES}

[1] D. E. Barrick and B. J. Lipa (1997), Evolution of bearing determination in HF current mapping radars, Oceanography, vol. 10, no. 2, pp. 72-75, 1997.

[2] B. J. Lipa and D. E. Barrick, Least-squares methods for the extraction of surface currents from CODAR crossed-loop data: Application at ARSLOE, IEEE J. Oceanic Engr., vol. OE-8, pp. 226-253, 1983.

[3] B. M. Emery and L. Washburn, "Comparison of surface current measurements in the Santa Barbara Channel: HF radar and moored current meters, Fall American Geophysical Meeting, EOS Transactions, AGU Vol. 79, No. 45, p. F393, 1998.

[4] D. C. Melton, Remote sensing and validation of surface currents from HF radar, Naval Postgraduate School Thesis, Monterey, CA, 66 pp., 1995.

[5] D. E. Barrick and B. J. Lipa, A Comparison of directionfinding and beam-forming in HF radar ocean surface current mapping, SBIR Phase I Final Report, Contract No. 50DKNA-5-00092 to National Oceanic \& Atmospheric Administration, CODAR Ocean Sensors, Ltd., Los Altos, CA, 1996. 